\title{
Agrotóxicos em água para consumo humano: uma abordagem de avaliação de risco e contribuição ao processo de atualização da legislação brasileira
}

\author{
Pesticides in drinking water: a risk assessment approach and contribution \\ to the Brazilian legislation updating process
}

\begin{abstract}
Maria de Lourdes Fernandes Neto
Mestre em Saneamento, Meio Ambiente e Recursos Hídricos pela Universidade Federal de Minas Gerais (UFMG). Doutoranda em Saúde Pública e Meio Ambiente pela Escola Nacional de Saúde Pública da Fundação Oswaldo Cruz (ENSP/Fiocruz). Consultora em Saúde Ambiental da Coordenação Geral de Vigilância em Saúde Ambiental da Secretaria de Vigilância em Saúde do Ministério da Saúde (CGVAM/SVS/MS)

Paula de Novaes Sarcinelli

Mestre em Farmacocinética e Metabolismo de Medicamentos pela Université de Paris XI (Paris-Sud), U.P. XI, França. Doutora em Biologia Celular e Molecular pela Fiocruz. Pesquisadora da Fiocruz
\end{abstract}

\section{Resumo}

O uso intensivo de agrotóxicos no país tem suscitado a preocupação de profissionais de diversos setores face aos riscos potenciais que essas substâncias trazem ao ambiente e aos seres humanos. Nesse contexto, a água para consumo humano pode ser uma importante forma de exposição. O padrão nacional de potabilidade da água é regulamentado pela Portaria do Ministério da Saúde n 518/2004 e contempla 22 agrotóxicos. O objetivo deste artigo é apresentar e discutir aspectos vinculados à atualidade do padrão de potabilidade brasileiro com relação à necessidade de inserção de agrotóxicos, e à definição de valores máximos permitidos (VMP). A estratégia da metodologia de avaliação de risco foi utilizada para a sistematização e análise das informações disponíveis na literatura. As discussões e apontamentos do trabalho poderão contribuir para a revisão da legislação nacional, cujo processo deverá se iniciar brevemente.

Palavras-chave: avaliação de risco; padrão de potabilidade; agrotóxicos.

\begin{abstract}
The intensive use of pesticides in the country has addressed the stakeholders concerns regarding the potential risks of these substances to the environment and to the human beings. In this context, the water for human intake can be an important form of exposure. The drinking water national standard is regulated by the Ministry of Health Act 518/2004, which considers 22 pesticides. The objective of this paper is to present and to discuss aspects of the current Brazilian standard of water potability, with regard to the necessity of insertion of pesticides and to the definition of maximum permitted values (VMP). The strategy of the methodology of risk assessment was used for the systematization and analysis of the available information in literature. The discussions and notes of the paper could contribute for the forthcoming national legislation review process.
\end{abstract}

Keywords: risk assessment; drinking water standards; pesticides.

\section{Introdução}

A maioria dos contaminantes químicos presentes em águas subterrâneas e superficiais está relacionada às fontes industriais e agrícolas. A variedade é enorme, com destaque para os agrotóxicos, compostos orgânicos voláteis e metais (HU; KIM, 1994). Agrotóxicos e afins são produtos e componentes de diferentes processos, e de uso na produção, armazenamento e beneficiamento na agricultura, pastagem, proteção de florestas e outros ambientes, para preservá-los da ação danosa de seres nocivos e, ainda, as substâncias e produtos usados como desfolhantes, dessecantes, estimuladores e inibidores do crescimento (BRASIL, 1989). Os agrotóxicos assumem caráter destacado enquanto contaminantes pela intensidade e, não raro, indiscriminação que caracterizam seu consumo no país. Sua presença nos mananciais pode trazer dificuldades para o tratamento da água em virtude da eventual necessidade de tecnologias mais complexas do que aquelas normalmente usadas para a potabilização. 
Dada a dinâmica dos agrotóxicos no ambiente e sua relevância no contexto da saúde das populações, a definição de diretivas e regulamentações governamentais acerca da produção, comercialização e uso desses produtos deve ser conduzida a partir de rigorosos aspectos. A $3^{a}$ edição dos Guias da Organização Mundial de Saúde (OMS) sobre qualidade da água para consumo humano aponta tais premissas para o estabelecimento de um valor guia para uma substância química (WORLD HEALTH ORGANIZATION, 2006): (a) há fortes evidências sobre a ocorrência da substância na água, assim como de sua toxicidade real ou potencial; (b) relevante significado internacional ou (c) a substância é parte integrante do Programa de Avaliação de Pesticidas da OMS (World Health Organization Pesticide Evaluation Scheme - WHOPES).

A OMS recomenda que, a despeito das substâncias químicas que representam risco à saúde humana e referidos valores guias apresentados em sua $3^{\text {a }}$ Edição, os países priorizem e incluam em suas normas as mais importantes segundo cada realidade. Nesse sentido, Bastos et al (2001) assinalam que a definição dos parâmetros de interesse e seus respectivos valores máximos permitidos (VMP), no padrão de potabilidade para as substâncias químicas implica na consideração de quesitos como: (I) análise das evidências epidemiológicas e toxicológicas e dos riscos à saúde associados às substâncias, essencialmente com base

Tabela 1 - Etapas constituintes da metodologia de avaliação de risco

\section{Identificação do perigo}

Compreende uma avaliação do conhecimento disponível e a descrição dos efeitos adversos à saúde, crônicos ou agudos, associados a determinado agente. Em termos da água utilizada para consumo humano, a existência de exploração agrícola na bacia de contribuição do manancial, descarga de efluentes de agroindústrias, falhas no tratamento da água, rupturas de rede de abastecimento são exemplos de risco.

\section{Avaliação da dose-resposta}

Após caracterização do perigo e identificação do agente associado, é preciso avaliar o potencial do agente em causar resposta em diversos níveis de exposição, assim como a sua extensão seus efeitos. Assim, é conduzida uma análise das relações entre a quantidade administrada/ absorvida do agente e as mudanças ocorridas no organismo, sistema ou população. A definição da dose que causa algum efeito adverso é estabelecida a partir de estudos experimentais, principalmente em animais, ou epidemiológicos.

\section{Avaliação da exposição}

Compreende a determinação do tamanho e caracterização da população exposta e a magnitude da exposição: definição da(s) rota(s), quantificação, duração e frequência da exposição. Algumas informações são importantes, como: produção e liberação das substâncias no ambiente, fatores relacionados ao movimento, persistência e degradação da substância; tamanho e distribuição das populações vulneráveis e informações sobre a ingestão do agente. Procura-se não apenas identificar o agente presente na água, mas quantificá-lo, além de determinar as circunstâncias envolvidas na exposição humana.

\section{Caracterização do risco}

Integra os resultados das etapas anteriores, gerando informações de natureza qualitativa e quantitativa. A partir do conhecimento da dose do agente (quantidade presente na água) e do consumo de água, determina-se, a partir de modelos matemáticos, o risco do agravo para uma ou mais exposições. Dessa forma, estima-se a magnitude do problema de saúde, considerando-se a variabilidade e a incerteza, com o intuito de se subsidiarem as estratégias de gerenciamento de risco.

Fonte: adaptado de United States Environmental Protection Agency (1990); Haas, Rose e Gerba (1999); WHO (1999; 2004); Bevilacqua et al (2002) apud Bastos et al (2003). em documentação da OMS, Environmental Protect Agency (EPA) e International Agency for Research on Cancer (Iarc); (II) potencial tóxico das substâncias que podem estar presentes na água naturalmente ou por contaminação; (III) intensidade de uso das substâncias no país (industrial, agrícola e no tratamento da água); (IV) possibilidade de obtenção de padrões analíticos e limitação de técnicas analíticas atualmente empregadas e (V) comparação dos valores guias da OMS, normas dos Estados Unidos da América, Canadá e Comunidade Europeia e os VMP definidos na legislação nacional em vigor.

A atual legislação brasileira de potabilidade de água, Portaria MS no 518/2004, regulamenta 54 substâncias químicas que representam riscos à saúde humana, dentre as quais 22 são agrotóxicos. A experiência internacional (com destaque para os Guias da OMS), que aborda e discute a inclusão de novas substâncias e o estabelecimento de seus respectivos VMP é utilizada, usualmente, como diretriz para a legislação brasileira (HELLER et al, 2005). Na última revisão da legislação (revisão da Portaria GM n 36/1990 e publicação da Portaria MS no 1469/2000, republicada em 2004, como Portaria MS $n^{\circ}$ 518), os VMP adotados foram, praticamente, os sugeridos pela OMS (ORGANIZACIÓN MUNDIAL DE LA SALUD, 1995). Assim, com a divulgação da 3a Edição dos Guias da OMS em 2004, e considerando-se o prazo previsto na Portaria MS no 518 para sua revisão (cinco anos), é importante que as autoridades de Saúde Pública do Brasil estejam atentas às novas diretivas expressas no documento, para a atualização da referida legislação.

O objetivo deste artigo é apresentar e discutir aspectos vinculados à atualidade do padrão de potabilidade nacional quanto à necessidade de inserção de agrotóxicos na norma e à definição de VMP, utilizando-se a estratégia da metodologia de avaliação de risco para a sistematização e análise das informações disponíveis na literatura.

\section{Metodologia de avaliação de risco e sua aplicação na determinação de VMP}

O termo risco é definido como a probabilidade de ocorrência de efeito adverso a um organismo, sistema ou população, causado sob circunstâncias específicas, devido à exposição a um agente (WORLD HEALTH ORGANIZATION, 2004). Segundo Guivant (2000), os estudos quantitativos sobre os riscos iniciaram seu desenvolvimento tendo por base disciplinas como toxicologia, epidemiologia, psicologia e engenharia a partir dos anos 1960. A avaliação de risco é definida como o processo que permite a caracterização quantitativa ou qualitativa e previsão/estimativa de efeitos adversos potenciais à saúde de determinada população, sistema ou organismo decorrentes da exposição a perigos de distintas naturezas (UNITED STATES ENVIRONMENTAL PROTECTION AGENCY, 1990; HAAS; ROSE; GERBA, 1999; WORLD HEALTH ORGANIZATION, 1999; 2004). O uso dessa metodologia pressupõe o desenvolvimento de suas quatro etapas constituintes, conforme apresentado na Tabela 1. 
Em termos da contaminação da água utilizada para consumo humano, o uso dos preceitos da avaliação de riscos é anterior à década de 1970, sendo que foi oficialmente instituída, como método, em 1983 pela National Academy of Science (NAS) dos EUA (HAAS; ROSE; GERBA, 1999; HACON; BARROCAS; SICILIANO, 2005). Segundo esses autores, tal metodologia, pioneira da NAS, teve seus princípios, processos e métodos refinados ao longo dos anos, notadamente pela Agência de Proteção Ambiental dos Estados Unidos (United States Environmental Protection Agency - Usepa) e, atualmente, tem sido bastante utilizada em diversas áreas do conhecimento. A avaliação de risco pode ser considerada parte de uma abordagem mais ampla, denominada Análise de Risco, e que compreende, ainda, o Gerenciamento (Risk Management) e a Comunicação do Risco (Risk Communication) (UNITED STATES DEPARTMENT OF AGRICULTURE, 2007).

Dentre algumas limitações apontadas a respeito do uso da metodologia de avaliação de risco, destacam-se (HAAS; ROSE; GERBA, 1999; UNITED STATES ENVIRONMENTAL PROTECTION AGENCY, 1990): (a) sensibilidade e limitações dos estudos epidemiológicos disponíveis; (b) extrapolação de dados obtidos a partir de estudos em animais para se estimarem efeitos à saúde humana, sobretudo quanto aos aspectos de suscetibilidade; (c) modelos matemáticos utilizados na extrapolação de altas doses para baixas doses de exposição e (d) manipulação das incertezas, nas estimativas. Outro ponto que merece destaque é que o ser humano pode estar exposto, ao mesmo tempo, a mais de uma substância ou à mistura de agentes químicos. Os efeitos dessa exposição múltipla podem ser distintos, de acordo com tipo e concentração do agente, via de exposição e características do indivíduo, como sexo, idade, dieta, estilo de vida, entre outros (PAUMGARTTEN, 1993). Além disso, e na maioria das vezes, alguns dos componentes da mistura são desconhecidos, as informações sobre a exposição não são confiáveis, ou variam ao longo do tempo, assim como os dados toxicológicos dos compostos conhecidos são limitados. No caso de misturas, a EPA recomenda procedimentos específicos para a avaliação de risco (UNITED STATES ENVIRONMENTAL PROTECTION AGENCY, 2000B; 2002).

O estabelecimento do padrão de potabilidade para substâncias químicas que representam risco à saúde é conduzido a partir da avaliação quantitativa do risco. O VMP para cada substância é, geralmente, estabelecido a partir de evidências toxicológicas ou epidemiológicas, que permitam estimar um Nível de Efeito Adverso Não Observado (No Observable Adverse Effect Level - NOAEL). As provas de toxicidade são usualmente obtidas em experimentos com animais. A partir desses estudos, definem-se níveis de exposição segura para os seres humanos, considerando-se variações inter e intraespécie (por exemplo, objetivando proteger populações mais sensíveis ou suscetíveis). Para tanto, aplica-se um fator de incerteza ao valor de NOAEL encontrado no estudo (WORLD HEALTH ORGANIZATION, 2004). A OMS ressalta que, quando possível, os valores de NOAEL devem ser obtidos a partir de estudos de longo prazo, preferivelmente envolvendo a ingestão de água. Entretanto, os valores de NOAEL encontrados em estudos desenvolvidos para curto prazo ou naqueles em que foram consideradas outras fontes de exposição também podem ser utilizados. Outra questão apontada é que na ausência do valor de NOAEL, pode-se utilizar o valor referente ao Menor Nível de Efeito Adverso Observado (Lowest Observed Adverse Effect Leavel - LOAEL), que indica a menor concentração ou nível de determinada substância, para a qual foi observado um efeito adverso à saúde. Nesse caso, deve-se aplicar um fator de incerteza adicional para o uso do LOAEL em substituição ao NOAEL (WORLD HEALTH ORGANIZATION, 2006). O nível de exposição segura é, então, a dose abaixo da qual as pessoas poderiam estar expostas sem que ocorressem danos à saúde, o que é comumente chamado de Ingestão Diária Aceitável (IDA) ou Ingestão Diária Tolerável (IDT). A IDA/IDT ${ }^{1}$ é uma estimativa da quantidade de determinada substância presente nos alimentos ou na água potável, expressa em função da massa corporal ( $\mathrm{mg} / \mathrm{kg}$ ou $\mu \mathrm{g} / \mathrm{kg})$, que se pode ingerir, diariamente, ao longo de toda a vida sem risco considerável para a saúde (WORLD HEALTH ORGANIZATION, 2006).

IDT/IDA $=$ NOAEL ou LOAEL FI Equação 1

$\frac{\mathrm{VMP}=\mathrm{IDT} \times \mathrm{mc} \times \mathrm{P}}{\mathrm{C}}$ Equação 2

onde:

FI: fator de incerteza; mc: massa corporal;

P: fração de IDT/IDA proveniente da água potável;

C: consumo diário de água.

A OMS adota os seguintes valores: $\mathrm{mc}=60 \mathrm{~kg}$ para adultos; $\mathrm{P}=10 \% ; \mathrm{C}=2 \mathrm{~L}$ para adultos.

A despeito das limitações apontadas por alguns autores, sobretudo quanto às incertezas e à abrangência, os preceitos da avaliação de risco encontram-se subjacentes no processo contínuo de formulação e atualização do padrão de potabilidade de países, como Canadá e Estados Unidos, e dos Guias da OMS (WORLD HEALTH ORGANIZATION, 2006; HEALTH CANADA, 2008; UNITED STATES ENVIRONMENTAL PROTECTION AGENCY, 2005A; 2005B).

\footnotetext{
${ }^{1}$ Embora correntemente utilizados como sinônimos, a OMS considera o termo IDT mais apropriado quando se trata da exposição aos contaminantes químicos que não seriam, normalmente, adicionados à água potável ou alimentos, pois assumiria o significado de permissibilidade e não aceitabilidade (WHO, 2006). Dessa forma, neste trabalho será utilizado o termo IDT.
} 


\section{Identificação do perigo e avaliação da relação dose-resposta}

\section{Agrotóxicos na legislação brasileira e em normas e critérios internacionais de qualidade da água para consumo humano}

A primeira legislação brasileira de potabilidade de água foi editada no final da década de 1970 (Portaria BSB n 56/1977) e contava com cerca de 40 parâmetros de qualidade de substâncias inorgânicas, agrotóxicos e parâmetros de aceitação para o consumo. Na passagem da Portaria BSB nº 56/1977 à Portaria no 36/1990, e mesmo à Portaria $n^{\circ} 1469 / 2000$, foram relativamente poucas as alterações em termos de número de substâncias inorgânicas que afetam a saúde e a aceitação para consumo. Entretanto, em relação às substâncias orgânicas e, sobretudo aos agrotóxicos, várias delas foram incorporadas à Portaria $n^{\circ} 36$ e à Portaria no 1469, (Portaria MS no 518), pois entre 1977 e 2000, diversas substâncias/princípios ativos ganharam mercado no país e ainda não eram contempladas na legislação (BASTOS, 2003). $\mathrm{O}$ atual padrão de potabilidade para substâncias químicas que representam risco à saúde é explicitado segundo o tipo (inorgânicas, orgânicas, agrotóxicos, desinfetantes e produtos secundários da desinfecção). Devido à sua relevância quanto ao uso no país e risco à saúde, os agrotóxicos (22 no total), foram considerados separadamente

Os valores-guia para as substâncias químicas, na $3^{\text {a }}$ Edição dos Guias da OMS, estão relacionados de acordo com a categoria de sua fonte de geração: a) ocorrência natural; b) fontes industriais e assentamentos humanos; c) utilizados na agricultura; d) utilizados no tratamento da água ou devido a materiais que entram em contato com a água; e) utilizados na água em programas de saúde pública e f) toxinas de cianobactérias. Em termos da categoria, são quatro as substâncias com valor-guia relacionado (Clorpirifós, Dicloro-DifenilTricloroetano - DDT - e metabólitos, Permetrina e Pyriproxyfen), embora a OMS enfatize que apenas a última seja recomendada para adição à água em campanhas de saúde pública no combate a vetores de doenças (WORLD HEALTH ORGANIZATION, 2006).

A definição de um padrão para contaminantes, nos Estados Unidos, obedece a um processo que leva em consideração: (a) ocorrência no ambiente; (b) fatores de exposição e de riscos à saúde da população geral e de grupos vulneráveis; (c) disponibilidade de métodos analíticos de detecção; (e) factibilidade técnica de atendimento e (f) impactos econômicos e de saúde pública (BASTOS et al, 2004). A regulação dos parâmetros ocorre, inicialmente, pela definição de um padrãometa não mandatório (Maximum Contaminat Level Goal - MCLG), pelo qual são levados em conta apenas aspectos de saúde. Normalmente, a definição do MCLG evolui para o estabelecimento de um Maximum Contaminant Level (MCL) ou VMP, mandatório. Esse VMP é estabelecido o mais próximo possível do MCLG, considerando-se a viabilidade técnico-econômica de atendimento e que, eventualmente, pode ser revisto com base em análises de custo-benefício. $\mathrm{O}$ atual padrão norteamericano para as substâncias químicas constitui-se de 171 substâncias orgânicas, 31 inorgânicas, além dos radionuclídeos (UNITED STATES ENVIRONMENTAL PROTECTION AGENCY, 2004).

Os valores-guia preconizados pela normativa canadense têm sido revisados desde 1996, quando foi publicada a $6^{\text {a }}$ edição dos guias para a água de consumo humano no país. Atualmente, são normatizados 78 parâmetros químicos, dentre os quais cerca de 30 são agrotóxicos (HEALTH CANADA, 2008). Em relação à Comunidade Europeia, os VMP que constavam na Diretiva de 1980 foram revisados em 1998, tendo os Guias da OMS como base assim como o conhecimento científico à época e o Princípio da Precaução². A Diretiva atual menciona que os parâmetros a serem adotados pelos países-membro devem ser baseados em questões de saúde pública e na metodologia de avaliação de riscos. Quanto aos agrotóxicos, a Diretiva considera os parâmetros 'Agrotóxico' e 'Agrotóxico total', cujos VMP são, respectivamente,

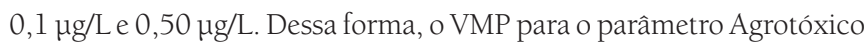
aplica-se, individualmente, para cada substância à exceção de Aldrin, Dieldrin, Heptacloro e Heptacloro epóxido, cujos VMP são o mesmo: $0,03 \mu \mathrm{g} / \mathrm{L}$. No caso do parâmetro Agrotóxico total, a soma das concentrações de todas as substâncias detectadas não deve ser superior a 0,50 g/L (MARQUES, 1996; COUNCIL DIRECTIVE, 1998).

Na Tabela 2, estão relacionados os agrotóxicos regulamentados pela atual legislação brasileira de potabilidade e os VMP correspondentes, preconizados pela OMS, Usepa e Health Canadá. Dentre os parâmetros dessa tabela, a legislação canadense é mais flexível em termos dos VMP quando comparada às demais normativas, sobretudo para o aldrin/dieldrin e metoxicloro, para os quais a norma canadense chega a ser 23 e 45 vezes superior, respectivamente. A última publicação relacionada ao padrão de potabilidade canadense indica que o VMP para o aldrin/dieldrin foi aprovado em 1994, ao passo que o VMP originalmente proposto para o metoxicloro, em 1986, foi revisto e mantido, em 2005, em $900 \mu \mathrm{g} / \mathrm{L}$ (HEALTH CANADA, 2008).

A OMS aponta que as substâncias bentazona, endossulfan, glifosato, heptacloro, heptacloro epóxido, hexaclorobenzeno e permetrina, por ocorrerem na água em concentrações abaixo das quais os efeitos tóxicos podem ser observados, não são consideradas, necessariamente, para a definição de valores-guia. Para o pentaclorofenol, foi atribuído um valorguia provisório em virtude de variações no metabolismo de animais, provados por experimentos, quando comparados aos seres humanos. Quanto ao propanil, a OMS enfatiza que é transformado rapidamente em metabólitos mais tóxicos e o estabelecimento de um valor-guia para os seus compostos é inapropriado. Além disso, as informações existentes são inadequadas para permitir a definição de valores guias para os metabólitos (WORLD HEALTH ORGANIZATION, 2006).

2 Princípio 15 da Declaração do Rio de Janeiro de 1992 segundo o qual a ausência de certeza científica absoluta não justifica postergar medidas eficazes para prevenir a degradação ambiental. 
Gomes, Spadatto, Lanchotte (2001) apontam que, ao contrário da normativa europeia, a legislação nacional é menos restritiva quanto aos VMP e contempla um número relativamente pequeno de agrotóxicos em relação à diversidade realmente utilizada no país. Da mesma forma, Sarcinelli et al (2005) enfatizam que embora a legislação brasileira contemple importantes agrotóxicos, sua amplitude ainda é muito deficiente. Segundo esses autores, tal deficiência, dentre outros motivos, caracteriza-se pelo fato de que a Portaria MS no 518/2004 não especifica alguns princípios ativos de relevância, como as classes dos organofosforados e carbamatos, largamente utilizados no país e de grande toxicidade.

\section{Risco à saúde humana (ingestão oral)}

O conhecimento sobre os efeitos, sobre a saúde, decorrentes da exposição às substâncias químicas, ocorre a partir de duas fontes principais de informação: estudos em seres humanos e estudos de toxicidade com animais. A fonte preferencial seria aquela relacionada aos estudos em grupos populacionais. Entretanto, o número de trabalhos para muitas substâncias é limitado, seja pela inexistência de informações quantitativas sobre os níveis de exposição, seja pela exposição concomitante a outros agentes. Os estudos em animais são mais frequentes, mas impõem limitações por incluírem relativamente poucos animais e, quase sempre, os exporem a concentrações elevadas. Essas limitações condicionam a utilização de fatores de incerteza, ao serem extrapolados dados de estudos em animais para humanos (WORLD HEALTH ORGANIZATION, 2006).

Os estudos para determinação da toxicidade objetivam identificar a natureza do dano causado e as faixas de doses sob as quais o efeito é produzido. A consideração inicial costuma ser a realização de um estudo de toxicidade aguda (dose única) para a substância em questão. Tais estudos de toxicidade aguda irão subsidiar o cálculo das doses que não serão letais, nos estudos de toxicidade de longa duração (UNITED STATES ENVIRONMENTAL PROTECTION AGENCY, 1990).

Faria, Fassa e Fachini (2007) discutiram aspectos relacionados às intoxicações por agrotóxicos no país e aos desafios para o desenvolvimento de estudos epidemiológicos. Algumas considerações foram apontadas no que tange às lacunas existentes de pesquisas epidemiológicas sobre o tema, sobretudo quanto aos efeitos crônicos. Nesse sentido, os autores ressaltam que, a despeito do crescimento quantitativo e qualitativo de estudos, incluindo aqueles que contemplam o ambiente e a questão alimentar, ainda há muito o que ser trabalhado, especialmente no que diz respeito às dificuldades encontradas tanto em caracterizar a exposição crônica, quanto em obter informações sobre os efeitos de longo termo associados.

Os efeitos sobre a saúde decorrentes do consumo de água contaminada por agrotóxicos variam segundo o princípio ativo envolvido. Dentre os problemas já identificados e publicados pela literatura internacional especializada, destacam-se (INTERNATIONAL AGENCY FOR RESEARCH ON CANCER, 2007; AGENCY FOR TOXIC SUBSTANCES AND DISEASE REGISTRY, 2007): (I) problemas no
Tabela 2 - Comparação entre os VMP dos agrotóxicos regulamentados pela Portaria MS $n^{0}$ 518/2004 e diferentes normatizações internacionais, em $\mu \mathrm{g} / \mathrm{L}$

\begin{tabular}{|c|c|c|c|c|}
\hline Parâmetro & $\begin{array}{l}\text { Portaria } \\
\text { MS n } n^{\circ} 518\end{array}$ & $\begin{array}{l}\text { Guias } \\
\text { OMS }^{(1)}\end{array}$ & $\mathrm{EPA}^{(1)}$ & $\begin{array}{l}\text { Health } \\
\text { Canadá(1) }\end{array}$ \\
\hline Alaclor & 20 & 20 & 2 & \\
\hline Aldrin/Dieldrin & 0,03 & 0,03 & & 0,7 \\
\hline Atrazina & 2 & 2 & 3 & 5 \\
\hline Bentazona & 300 & & & \\
\hline Clordano (isômeros) & 0,2 & 0,2 & 2 & \\
\hline $2,4 \mathrm{D}$ & 30 & 30 & 70 & 100 \\
\hline DDT (isômeros) & 2 & 1 & & \\
\hline Endossulfan & 20 & & & \\
\hline Endrin & 0,6 & 0,6 & 2 & \\
\hline Glifosato & 500 & & 700 & 280 \\
\hline $\begin{array}{l}\text { Heptacloro e } \\
\text { heptacloro epóxido }\end{array}$ & 0,03 & & 0,4 e 0,2 & \\
\hline Hexaclorobenzeno & 1 & & 1 & \\
\hline Lindano $(\mathrm{g}-\mathrm{BHC})$ & 2 & 2 & 0,2 & \\
\hline Metolacloro & 10 & 10 & & 50 \\
\hline Metoxicloro & 20 & 20 & 40 & 900 \\
\hline Molinato & 6 & 6 & & \\
\hline Pendimetalina & 20 & 20 & & \\
\hline Pentaclorofenol & 9 & 9 & 1 & 60 \\
\hline Permetrina & 20 & $300^{(2)}$ & & \\
\hline Propanil & 20 & & & \\
\hline Simazina & 2 & 2 & 4 & 10 \\
\hline Trifluralina & 20 & 20 & & 45 \\
\hline
\end{tabular}

Fonte: Brasil (2005); Health Canada (2008); United States Environmental Protection Agency (2004); WHO (2006)

(1) para os campos não preenchidos, não há previsão de valor guia ou VMP; (2) quando utilizada na água como larvicida.

fígado e no sistema nervoso central, como dores de cabeça, tonturas, irritabilidade, movimentos musculares involuntários; (II) problemas com os sistemas cardiovascular e reprodutivo, com algumas evidências de desregulação endócrina e (III) problemas nos olhos, rins, baço, anemia e aumento do risco de desenvolver câncer.

\section{Caracterização dos agrotóxicos normalizados pela Portaria MS n ${ }^{\circ}$ 518/2004}

As substâncias regulamentadas pela Portaria MS no 518/2004 são descritas na Tabela 3 segundo sua classificação e principais características. A maioria das substâncias é da classe dos herbicidas. Quanto à classificação toxicológica, o predomínio ocorre para substâncias da Classe III (medianamente tóxico), seguido da Classe I (extremamente tóxico). Dentre essas substâncias, várias não possuem autorização de uso no Brasil de modo que a monografia do ingrediente ativo não está disponibilizada pela Agência Nacional de Vigilância Sanitária (Anvisa) ou têm uso restrito a algumas culturas ou como preservantes de madeiras.

\section{Avaliação da exposição}

\section{Ocorrência de agrotóxicos em mananciais de abastecimento e em água distribuída para consumo humano no Brasil}

O Brasil é um dos maiores consumidores mundiais de agrotóxicos e, a despeito da existência de regulamentações quanto à comercialização 
Tabela 3 - Caracterização dos agrotóxicos regulados pela Portaria MS nº 518/2004

\begin{tabular}{|c|c|c|c|c|c|c|c|c|c|c|c|}
\hline \multirow[t]{2}{*}{ Agrotóxico } & \multirow[t]{2}{*}{ Classe $^{(1)}$} & \multirow{2}{*}{$\begin{array}{l}\text { Classificação } \\
\text { toxicológica }\end{array}$} & \multirow{2}{*}{$\begin{array}{c}\text { Índice } \\
\text { Anvisa }^{(3)}\end{array}$} & \multicolumn{2}{|c|}{$\begin{array}{l}\text { Solubilidade } \\
\text { em água(4) }\end{array}$} & \multicolumn{2}{|c|}{$\begin{array}{l}\text { Persistência } \\
\text { no Ambiente }{ }^{(5)}\end{array}$} & \multicolumn{2}{|c|}{$\begin{array}{c}\text { Potencial para } \\
\text { bioacumulação(6) }\end{array}$} & \multicolumn{2}{|c|}{$\begin{array}{c}\text { Potencial para } \\
\text { adsorção no solo }\end{array}$} \\
\hline & & & & Sim & Não & Sim & Não & Sim & Não & Sim & Não \\
\hline Alaclor & $\mathrm{H}$ & III & A06 & $\mathrm{X}$ & & & & & & & \\
\hline Aldrin e Dieldrin & I, A & 1 & - & & $\mathrm{x}$ & $x$ & & $\mathrm{x}$ & & $\mathrm{X}$ & \\
\hline Atrazina & $\mathrm{H}$ & III & A14 & $\mathrm{x}$ & & $X$ & & & $\mathrm{x}$ & & \\
\hline Bentazona & $\mathrm{H}$ & III & B03 & & & & $\mathrm{x}$ & & & & $\mathrm{x}$ \\
\hline Clordano (isômeros) & I & I & - & & $\mathrm{x}$ & $\mathrm{X}$ & & $\mathrm{x}$ & & $\mathrm{x}$ & \\
\hline $2,4 \mathrm{D}$ & $\mathrm{H}$ & I & D27 & $\mathrm{x}$ & & $x$ & & & $\mathrm{x}$ & & \\
\hline DDT (isômeros) & I & I & - & & $\mathrm{x}$ & $\mathrm{X}$ & & $\mathrm{X}$ & & & \\
\hline Endossulfan & A, I, Fo & I & E02 & & $\mathrm{x}$ & & & $x$ & & $x$ & \\
\hline Endrin & 1 & 1 & - & & $\mathrm{x}$ & $\mathrm{X}$ & & & & & \\
\hline Glifosato & $\mathrm{H}$ & IV & G01 & $\mathrm{x}$ & & & $\mathrm{x}$ & & $\mathrm{x}$ & $\mathrm{X}$ & \\
\hline $\begin{array}{l}\text { Heptacloro e } \\
\text { heptacloro epóxido }\end{array}$ & 1 & 1 & - & & $\mathrm{x}$ & $\mathrm{X}$ & & $\mathrm{X}$ & & & \\
\hline Hexaclorobenzeno & $\mathrm{Fu}$ & 1 & - & & $\mathrm{x}$ & $\mathrm{x}$ & & $\mathrm{x}$ & & & \\
\hline Lindano $(\gamma-\mathrm{BHC})$ & 1 & II & L01 & & $X$ & & & & & & \\
\hline Metolacloro & $\mathrm{H}$ & III & M16 & $\mathrm{x}$ & & & & $x$ & & $\mathrm{x}$ & \\
\hline Metoxicloro & I & III & M18 & & $\mathrm{x}$ & $\mathrm{x}$ & & $X$ & & & \\
\hline Molinato & $\mathrm{H}$ & II & M21 & & & & $x$ & & & & \\
\hline Pendimetalina & $\mathrm{H}$ & III & P05 & & & $x$ & & & & $\mathrm{X}$ & \\
\hline Pentaclorofenol & $\mathrm{Fu}$ & I & P44 & $\mathrm{x}$ & & $\mathrm{x}$ & & & & & \\
\hline Permetrina & I, Fo & III & P06 & $\mathrm{x}$ & & & & & & & \\
\hline Propanil & $\mathrm{H}$ & III & P16 & & & & $\mathrm{X}$ & & & & \\
\hline Simazina & $\mathrm{H}$ & III & $\mathrm{S} 03$ & & & $\mathrm{X}$ & & & & $\mathrm{X}$ & \\
\hline Trifluralina & $\mathrm{H}$ & III & T24 & & $x$ & & & $x$ & & $x$ & \\
\hline
\end{tabular}

Fonte: adaptado de Larini (1997); United States Environmental Protection Agency (2000A); Bastos (2006); Health Canada (2009); International Programme on Safety Chemical (2007); WHO (1994; 1999); Agência Nacional de Vigilância Sanitária (2007A; 2007B); Agency for Toxic Substances and Disease Registry (2007).

(1) classe: A: Acaricida; Fo: Formicida; Fu: Fungicida; H: Herbicida; I: Inseticida; (2) classificação toxicológica: Classe I: extremamente tóxico; Classe II: altamente tóxico; Classe III: medianamente tóxico; Classe IV: pouco tóxico; ${ }^{(3)}$ código da monografia, segundo a Anvisa; ${ }^{(4)}$ solubilidade em água: capacidade do agrotóxico em se dissolver em uma quantidade padrão de água; (5) persistência no ambiente: propriedade relacionada à degradação do agrotóxico no solo ou na água; ${ }^{(6)}$ potencial para bioacumulação: capacidade do agrotóxico em se acumular nos organismos vivos (vegetais ou animais); ${ }^{(7)}$ potencial para adsorção no solo: propriedade do agrotóxico em interagir com a superfície das partículas do solo.

e uso, os riscos da exposição humana a esses contaminantes (presentes em diferentes compartimentos ambientais) são uma realidade. $\mathrm{Na}$ Figura 1, é apresentado o volume de vendas de agrotóxicos (em milhões R\$) para o Brasil, nos anos 2007 (janeiro a outubro) e 2008, indicando expressiva participação dos herbicidas nesse volume de vendas, seguidos pelos fungicidas, para os dois anos.

Quanto aos estudos sobre mudanças espacial e temporal na qualidade das águas, devido aos agrotóxicos, Marques (2005) afirma que ainda são escassos no país. A autora destaca que, apesar da boa estruturação do sistema de monitoramento das águas interiores do estado de São Paulo, a Companhia de Tecnologia de Saneamento Ambiental de São Paulo (Cetesb) não privilegia os principais agrotóxicos/princípios ativos, comercializados. Por outro lado, o monitoramento de

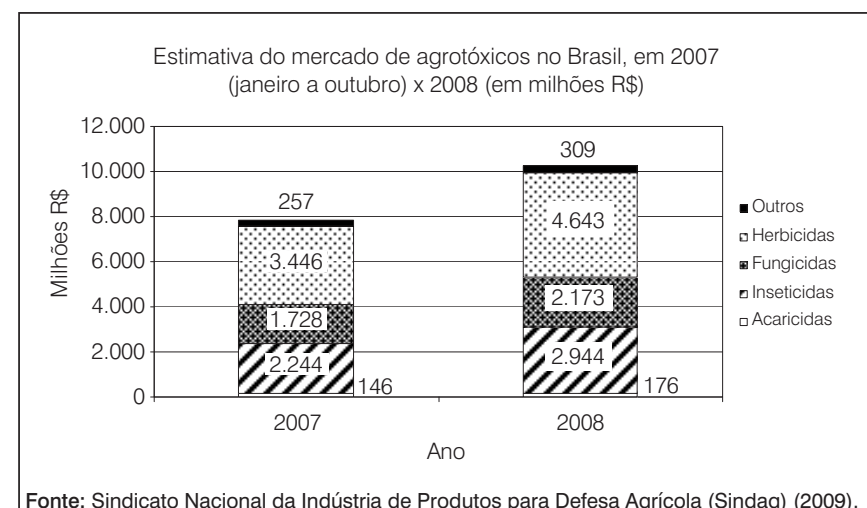

Figura 1 - Comercialização de agrotóxicos, no Brasil, nos anos de 2007 e 2008 agrotóxicos em águas destinadas ao consumo humano, por parte dos prestadores de serviços de abastecimento e pelo setor saúde, ainda é tímido, embora essa atividade esteja definida na Portaria MS n ${ }^{\circ}$ 518/2004, com frequência mínima semestral.

$\mathrm{Na}$ Tabela 4, são ilustradas a detecção e a estimativa de agrotóxicos em águas brutas e tratadas segundo trabalhos publicados no país entre os anos de 2000 e 2007. Além da relativa abrangência espacial, os dados da Tabela indicam uma diversidade considerável de substâncias identificadas nas águas utilizadas para consumo humano.

\section{Caracterização do risco}

\section{Caracterização da exposição e contribuição relativa ao consumo de água}

A exposição humana aos agrotóxicos ocorre segundo diferentes rotas, o que dependerá de cada circunstância. Em algumas dessas, os indivíduos podem ser expostos por mais de uma via ao mesmo tempo, o que configura uma exposição múltipla. Assim, por exemplo, um trabalhador rural pode ser exposto tanto durante a aplicação do agrotóxico, em dada cultura, quanto pelo consumo de alimentos ou água contaminados. Da mesma forma, populações que moram próximas a áreas cultivadas com agrotóxicos podem consumir água ou alimentos contaminados, bem como inalar a substância que eventualmente esteja no ar. Além disso, um mesmo indivíduo pode ser exposto a mais de um tipo de agrotóxico, ainda que segundo uma única rota, configurandose, também, uma situação preocupante de exposição. 
Tabela 4 - Resultados da pesquisa ou estimativa da presença de agrotóxicos em amostras de água para consumo humano, Brasil (2000 a 2007)

\begin{tabular}{|c|c|c|c|c|}
\hline \multirow{2}{*}{ Substâncias estudadas/princípios ativos } & \multicolumn{2}{|c|}{ Tipo manancial } & \multirow{2}{*}{ Região de interesse } & \multirow{2}{*}{ Fonte } \\
\hline & Sup..$^{(1)}$ & Sub. (2) & & \\
\hline $\begin{array}{l}\text { Metomil, maneb, triadimefon, atrazina, metribuzina, } \\
\text { simazina, clorimuron etil, flumetsulan, fomesafen, } \\
\text { glifosato, imazaquin, imazetapir e metolaclor etc. }\end{array}$ & $\mathrm{x}$ & $\mathrm{x}$ & Primavera do Leste (MT) & (3) \\
\hline Herbicida Tebuthiuron & & $\mathrm{x}$ & Microbacia do córrego Espraiado, Ribeirão Preto (SP) & (4) \\
\hline $\begin{array}{l}\text { Alfa-Endossulfan, Beta-Endossulfan, 2,4D, Sulfato de } \\
\text { Endossulfan, Glifosato, Tetradifon e Triclorfon. }\end{array}$ & $\mathrm{x}$ & $\mathrm{x}$ & Nordeste brasileiro & $(5)$ \\
\hline $\begin{array}{l}\text { Organofosforados, benzimidazóis, carbamatos, } \\
\text { piretroides e compostos clorados }\end{array}$ & $\mathrm{x}$ & $x$ & Petrolina (PE) e Juazeiro (BA) & (6) \\
\hline Organoclorados & $\mathrm{x}$ & & Bauru (SP) & $(7)$ \\
\hline $\begin{array}{l}\text { Aldicarbe, carbofurano e carbaril, simazina e atrazina } \\
\text { e trifluralina }\end{array}$ & $\mathrm{x}$ & $x$ & Região do rio Ribeira de Iguape (SP) & (8) \\
\hline Organoclorados, organofosforados e piretroides & $\mathrm{X}$ & & $\begin{array}{l}\text { Principais bacias hidrográficas de } \\
\text { MG, PR, SC, RS, MS, MT, RJ }\end{array}$ & (9) \\
\hline Princípios ativos: imidacloprid, atrazina, clomazone & $\mathrm{x}$ & & Agudos (RS) & (10) \\
\hline Diversos organoclorados e metais & $\mathrm{x}$ & & Região central do Estado de São Paulo & (11) \\
\hline Organofosforados e carbamatos & $\mathrm{x}$ & $\mathrm{x}$ & Paty do Alferes (RJ) & (12) \\
\hline Herbicidas: clomazone, propanil e quinclorac & $\mathrm{X}$ & & Rio Grande do Sul & (13) \\
\hline
\end{tabular}

Tabela 5 - Contribuição relativa ao consumo de água, em termos da IDT

\begin{tabular}{|c|c|c|c|c|c|}
\hline Parâmetro & VMP $(\mu \mathrm{g} / \mathrm{L})$ & IDT Anvisa (mg/kg mc) & IDT adulto(1) (mg/dia) & $\mathrm{IDMT}^{(2)}(\mathrm{mg} / \mathrm{dia})$ & IDMT/IDT ${ }^{(3)}(\%)$ \\
\hline Bentazona & 300 & 0,1 & 6,0 & 0,6 & 10,0 \\
\hline $2,4 \mathrm{D}$ & 30 & 0,01 & 0,60 & 0,06 & 10,0 \\
\hline Endossulfan & 20 & 0,006 & 0,36 & 0,04 & 11,1 \\
\hline Glifosato $^{(4)}$ & 500 & 0,042 & 2,52 & 1 & 39,7 \\
\hline Permetrina & 20 & 0,05 & 3,0 & 0,04 & 1,3 \\
\hline
\end{tabular}

Fonte: elaborado a partir de Brasil (2005) e Agência Nacional de Vigilância Sanitária (2007).

(1) considerando-se $60 \mathrm{~kg}$ de massa corporal, IDT adulto = IDT $(\mathrm{mg} / \mathrm{kg} \mathrm{mc})$ × $60(\mathrm{~kg}) ;{ }^{(2)}$ ingestão Diária Máxima Teórica para um consumo diário de $2 \mathrm{~L}$ de água = VMP (mg/L) x 2 L; (3) percentual do VMP atribuído ao consumo de água; (4) Bastos (2006).

A definição de VMP para substâncias químicas ocorre a partir da estimativa da IDT, que inclui a contribuição relativa ao consumo de água, além de outras possíveis formas de exposição, como o contato dérmico ou inalação, e a ingestão de alimentos provenientes de solo contaminado. Como exemplos, a Agency for Toxic Substances and Disease Registry (AGENCY FOR TOXIC SUBSTANCES AND DISEASE REGISTRY, 2007) afirma que em virtude de ser praticamente insolúvel em água, o hexaclorobezeno, em geral, não está presente na água de consumo humano em concentrações elevadas e essa exposição é, portanto, limitada. Estudos apontam a seguinte exposição média anual: alimentos (1,0 $\mu \mathrm{g} / \mathrm{kg}) ; \operatorname{ar}(0,01 \mu \mathrm{g} / \mathrm{kg})$; água $(0,00085 \mu \mathrm{g} / \mathrm{kg})$. Por outro lado, a exposição ao pentaclorofenol por ingestão de alimentos contaminados é considerada limitada. As estimativas de exposição diária, para um adulto com $70 \mathrm{~kg}$, são: alimentos (0,0105 mg); água $(0,021 \mathrm{mg}) ;$ ar (0,063 mg). A Anvisa estabelece a IDT para cinco dos 22 agrotóxicos relacionados pela Portaria MS no 518/2004: bentazona, 2,4D, endossulfan, glifosato e permetrina. Na Tabela 5, é apresentado um exercício semelhante ao efetuado por Bastos (2006), quanto à contribuição do VMP, correspondente ao consumo de água, em termos percentuais do valor total da IDT, para tais substâncias.

Do exposto na Tabela 4, depreende-se que sob a ótica da avaliação de risco, os VMP para o glifosato e endossulfan, na água de consumo humano, correspondem a cerca de 40 e 11\%, respectivamente, do valor total definido pela Anvisa para a IDT dessas substâncias. Os parâmetros bentazona e 2,4D respondem, ambos, por 10\% da IDT definida pela Anvisa, ao passo que o percentual atribuído ao VMP da permetrina é de cerca de $1 \%$. Tais valores indicam a contribuição máxima esperada devido à ingestão de água contendo as substâncias químicas relacionadas e apontam para a necessidade de que sejam averiguadas o quão representativas seriam essas contribuições, em termos de outras possíveis fontes de exposição, a exemplo dos alimentos, da inalação e do contato dérmico. Questões como: "o percentual máximo atribuído à exposição ao glifosato, por ingestão de água, por exemplo, pode ser considerado como de baixo risco, implicando em uma 'segurança' para o atual VMP dessa substância, na Portaria MS no 518/2004?" devem ser objeto de reflexão.

\section{Considerações finais}

Os 22 agrotóxicos contemplados na legislação nacional em vigor foram aqueles considerados mais relevantes, sobretudo em termos da intensidade de uso no país na época da revisão da Portaria no 36/1990. Os VMP correspondentes são praticamente os mesmos preconizados pela segunda edição dos Guias da OMS, de 1993. Ao se efetuar um paralelo entre os agrotóxicos da Portaria MS no 518/2004 e seus respectivos VMP com as diferentes normativas internacionais, observa-se que as substâncias bentazona, endossulfan e propanil não são consideradas parâmetros regulados nos Estados Unidos e Canadá, tampouco possuem valor-guia assinalado pela OMS em sua terceira edição. Além disso, algumas substâncias cuja produção/utilização foi suspensa ou restringida também não possuem especificação de VMP em algumas das normativas referenciadas, sob o argumento de não serem mais utilizadas. Dentre essas substâncias, destacam-se o aldrin/dieldrin, DDT, endrin, heptacloro 
e hexaclorobenzeno, que figuram entre os doze Poluentes Orgânicos Persistentes (POP) elencados pela Convenção de Estocolmo, em 2001, para proibição de produção e uso em função dos danos à saúde, evidenciados pela comunidade científica internacional (UNITED NATIONS ENVIRONMENT PROGRAMME CHEMICALS, 2001). Entretanto, tais substâncias são, além de tóxicas para os seres vivos, reconhecidamente persistentes no ambiente e apresentam potencial para bioacumulação, o que pressupõe a necessidade de avaliação quanto à pertinência de mantê-las em programas de monitoramento ambiental e, por conseguinte, no estabelecimento do padrão de potabilidade.

Os estudos, citados neste trabalho, sobre a presença de agrotóxicos em águas destinadas ao consumo humano, reforçam a assertiva de que o processo de revisão da legislação nacional contemple uma avaliação criteriosa que inclua, dentre outros, o diagnóstico dos princípios ativos mais utilizados e o perfil de uso dessas substâncias, pois podem ter seu uso centralizado em culturas e regiões específicas. Esse levantamento torna-se especialmente relevante ao se considerarem as características intrínsecas às substâncias (como persistência no ambiente, lixiviação e potencial para bioacumulação, dentre outras) e especificidades inerentes aos compartimentos ambientais (tipo de solo, $\mathrm{pH}$ e temperatura do solo e da água, dentre outras). Além disso, as técnicas necessárias à remoção de contaminantes orgânicos em água correspondem a tecnologias pouco comuns à maioria das Estações de Tratamento de água (ETA) convencionais, como adsorção em carvão ativado e filtração por membranas (osmose reversa e nanofiltração), o que evidencia o risco de que tais substâncias podem passar incólumes pelos processos de tratamento, colocando em risco as populações consumidoras.

Dentre as considerações necessárias à definição de VMP para agrotóxicos estão os resultados de estudos toxicológicos e epidemiológicos e fatores de incerteza para variações intra e interespécies. Além disso, são atribuídos valores de referência para o volume de água ingerido diariamente e a massa corporal média do indivíduo. Todas essas informações trazem consigo alguma possibilidade de distanciamento da realidade, em virtude das condições de contorno consideradas e, portanto, das incertezas associadas. Esse distanciamento torna-se ainda mais significativo quando tais informações são consideradas da mesma maneira para contextos distintos, como para países em que as características socioambientais divergem, em muito, daqueles nos quais foram obtidas as informações originalmente. Os valores normalmente atribuídos aos fatores de incerteza, volume de água consumido diariamente e a massa corporal, nos cálculos mencionados, são os mais adequados à realidade nacional? A realização de estudos específicos para o país, considerando-se as peculiaridades nacionais relacionadas ao consumo de agrotóxicos e à sua população talvez aponte para uma negativa ao questionamento. A necessidade de que mais estudos sejam conduzidos no país é reforçada pelo reconhecimento de que o Brasil dispõe de poucas informações sistematizadas quanto à identificação de contaminantes químicos em ambientes aquáticos e quanto às relações entre esses contaminantes e de efeitos adversos à saúde humana, em estudos toxicológicos e epidemiológicos nacionais. Tais lacunas do conhecimento precisam ser preenchidas, em especial, ao se considerar o caráter assumido pela água contaminada por organismos patogênicos em detrimento dos efeitos causados por agentes químicos. Enquanto os riscos causados pelos agentes patogênicos constituem problemas imediatos, agudos e por vezes associados a surtos de grandes proporções, os riscos químicos configuram, normalmente, um problema de longo prazo, cujos efeitos crônicos, muitas vezes, são de difícil detecção. Entretanto, a despeito da recomendação da OMS de que a garantia da qualidade microbiológica da água seja prioridade, as autoridades de saúde pública devem estar atentas e trabalhar, também, em prol da garantia da qualidade química da água para consumo humano.

A partir do exposto, algumas ações devem ser priorizadas ao se pensar em uma revisão da legislação nacional: a) sistematização e análise de informações disponíveis na literatura nacional e junto aos atores correspondentes, sobre comercialização e intensidade de uso de agrotóxicos no país; b) desenvolvimento de estudos relacionados à detecção e estimativa da presença de agrotóxicos em mananciais e em água distribuída para consumo humano; c) revisão de literatura sobre estudos epidemiológicos e toxicológicos dos princípios ativos mais utilizados no país; d) definição de critérios para priorizar os agrotóxicos mais relevantes, em termos de definição de VMP, inclusive contemplando a pertinência de manutenção dos atuais princípios ativos contemplados na legislação; e) desenvolvimento de estudos considerando condições de contorno diferentes daquelas utilizadas em outros países, quanto à definição da IDT para princípios ativos mais relevantes; f) a partir de uma abordagem de gerenciamento de riscos, avaliar a viabilidade técnica e financeira de remoção de agrotóxicos, tendo em vista as diferentes tecnologias disponíveis para o tratamento de água; g) sistematizar e avaliar as possibilidades analíticas de detecção de agrotóxicos em água quanto aos limites de detecção dos principais métodos e viabilidade técnica para incorporação das metodologias em larga escala pelos prestadores de serviço de abastecimento de água e laboratórios de saúde pública.

\section{Referências}

AGÊNCIANACIONAL DE VIGILÂNCIA SANITÁRIA. Monografias de produtos agrotóxicos. Atualizado em 15/fev./2007. Disponível em: http://uww.anvisa. gov.br/toxicologia/monografias/index.htm. Acesso em 23 mar. 2007A.
Sistema de Informações sobre Agrotóxicos - SIA. Disponível em: www4.anvisa.gov.br/agrosia/asp/default.asp. Acesso em 16 abr. 2007B. 
AGENCY FOR TOXIC SUBSTANCES AND DISEASE REGISTRY. Toxicological profile information sheet. Departament of health and human services. Disponível em: http://www.atsdr.cdc.gov/toxprofiles. Acesso em: 14 abr. 2007.

BASTOS, R.K.X. Glifosato: valor máximo permitido na água para consumo humano. Parecer Técnico. Ministério da Saúde/Secretaria de Vigilância em Saúde, maio de 2006.

Controle e vigilância da qualidade da água para consumo humano: evolução da legislação brasileira. In: CONGRESSO REGIONAL DE ENGENHARIA SANITÁRIA E AMBIENTAL DA $4^{a}$ REGIÃO DA AIDIS, CONE SUL, 4, 2003, São Paulo. Anais... Rio de Janeiro: ABES, 2003. (CD-ROM)

BASTOS, R.K.X. et al. Legislação sobre controle e vigilância da qualidade da água para consumo humano: a experiência brasileira comparada à panamericana. In: CONGRESSO INTERAMERICANO DE INGENIERÍA SANITARIA Y AMBIENTAL - AIDIS, 29, 2004, San Juan, Porto Rico. Anais... San Juan: Aidis, 2004. (CD-ROM).

Revisão da Portaria no 36 GM/90. Premissas e princípios norteadores. In: CONGRESSO BRASILEIRO DE ENGENHARIA SANITÁRIA E AMBIENTAL, 21, 2001, João Pessoa. Anais... Rio de Janeiro: ABES, 2001. (CD-ROM).

BEVILACQUA, P.D. et al. Densidades de Giardia e Cryptosporidium em mananciais de abastecimento de água e prevalência de giardíase: usos e aplicações do modelo teórico de avaliação de risco. In: CONGRESSO INTERAMERICANO DE ENGENHARIA SANITÁRIA E AMBIENTAL, 28, 2002, Cancún, México: Anais... Cidade do México: Associação Interamericana de Engenharia Sanitária e Ambiental, 2002. (CD-ROM).

BORTOLUZZI, E.C. et al. Contaminação de águas superficiais por agrotóxicos em função do uso do solo numa microbacia hidrográfica de Agudo, RS. Revista Brasileira de Engenharia Agrícola e Ambiental, v. 10, n. 4, p. 881-887, 2006.

BRASIL. Ministério da Saúde. Portaria MS no 518, de 25 de março de 2004. Estabelece os procedimentos e responsabilidades relativos ao controle e vigilância da qualidade da água para consumo humano e seu padrão de potabilidade, e dá outras providências. Brasília, DF: Ministério da Saúde, 2005. (Série E, Legislação em Saúde).

Lei $n^{\circ}$ 7.802, de 11 de julho de 1989. Dispõe sobre a pesquisa, a experimentação, a produção, a embalagem e rotulagem, o transporte, o armazenamento, a comercialização, a propaganda comercial, a utilização, a importação, a exportação, o destino final dos resíduos e embalagens, o registro, a classificação, o controle, a inspeção e a fiscalização de agrotóxicos, seus componentes e afins, e dá outras providências. Diário Oficial da União. Poder Executivo. Brasília, DF: Congresso Nacional, 1989

BRITO, N.M. et al. Risco de contaminação de águas por pesticidas aplicados em plantações de eucaliptos e coqueiros: análise preliminar. Pesticidas: Revista de Ecotoxicologia e Meio Ambiente, Curitiba, v. 11, p. 93-104, jan./dez. 2001.

CORBI, J.J. et al. Diagnóstico ambiental de metais e organolclorados em córregos adjacentes a áreas de cultivo de cana-de-açúcar (Estado de São Paulo, Brasil). Química Nova, v. 29, n. 1, p. 61-65, 2006
COUNCIL DIRECTIVE. Quality of water intend for human consumption. Jornal Oficial das Comunidades Europeias, 1998. Disponível em: http:// eur-lex.europa.eu/LexUriServ/LexUriServ.do?uri=OJ:L:1998:330:0032:0 054:EN:PDF. Acesso em: 30 jan. 2009.

DORES, E.F.G.C.; FREIRE, E.M.L. Contaminação do ambiente aquático por pesticidas. Estudo de caso: águas usadas para consumo humano em Primavera do Leste, Mato Grosso - Análise Preliminar. Química Nova, v. 24, n. 1, p. 27-36, 2001.

FARIA, N.M.X.; FASSA, A.G.; FACCHINI, L.A. Intoxicação por agrotóxicos no Brasil. Os sistemas oficiais de informação e desafios para a realização de estudos epidemiológicos. Ciência e Saúde Coletiva, Abrasco, v. 12. n. 1, p. 25-36, jan./mar. 2007.

FERRACINI, V.L. et al. Análise de risco de contaminação das águas subterrâneas e superficiais da região de Petrolina (PE) e Juazeiro (BA). Pesticidas: Revista de Ecotoxicologia e Meio Ambiente, Curitiba, v. 11, p. 1-16, jan./dez. 2001.

GOMES, M.A.F.; SPADOTTO, C.A.; LANCHOTTE, V.L. Ocorrência do herbicida Tebuthiuron na água subterrânea da microbacia do Córrego Espraiado, Ribeirão Preto, SP. Pesticidas: Riscos Ecotoxicológicos e Meio Ambiente, Curitiba, v. 11, p. 65-76, jan./dez. 2001.

GUIVANT, J.S. Reflexividade na sociedade de risco: conflitos entre leigos e peritos sobre os agrotóxicos. In: HERCULANO, S. (Org.). Qualidade de vida e riscos ambientais. Niterói: UFF, 2000. p. 281-303.

HAAS, C.N.; ROSE, J.B.; GERBA, C.P. Quantitative microbial risk assessment. USA: John Wiley \& Sons Inc., 1999.

HACON, S.; BARROCAS, P.; SICILIANO, S. Avaliação de risco para a saúde humana: uma contribuição para a gestão integrada de saúde e ambiente. Cadernos de Saúde Coletiva, Rio de Janeiro, v. 13, n. 4, p. 811-836, 2005.

HEALTH CANADA. Water Quality: reports and publications. Guidelines for Canadian drinking water quality. Chemical/Physical Parameters. Disponível em: http://www.hc-sc.gc.ca/ewh-semt/pubs/water-eau/indexeng.php\#tech_doc. Acesso em: 30 jan. 2009.

Guidelines for Canadian drinking water quality. Summary Table. Disponível em: http://www.hc-sc.gc.ca/ewh-semt/alt formats/hecssesc/pdf/pubs/water-eau/sum_guide-res_recom/summary-sommaireeng.pdf. 2008. Acesso em: 29 jan. 2009.

HELLER, L. et al. Terceira edição do guias da Organização mundial da Saúde: que impacto esperar na Portaria n 518/2004? In: CONGRESSO BRASILEIRO DE ENGENHARIA SANITÁRIA E AMBIENTAL, 23, 2005, Campo Grande, MS. Anais... Rio de Janeiro: ABES, 2005. (CD-ROM).

HU, H.; KIM, N. K. Drinking-water pollution and human health. In: CHIVIAN, E. et al. (Ed.). Critical condition: human health and the environment. 2. Ed. EUA: MIT Press, 1994. p. 31-45.

INTERNATIONAL AGENCY FOR RESEARCH ON CANCER. Complete list of agents evaluated and their classification. Disponível em: http:// monographs.iarc.fr/ENG/Classification/index.php. Acesso em: 7 mai. 2007. 
INTERNATIONAL PROGRAMME ON SAFETY CHEMICAL. Chemical safety information from intergovernmental organizations. Disponível em: www.inchem.org. Acesso em: 2 abr. 2007.

LARINI, L. Toxicologia. 3. Ed. São Paulo: Manole, 1997.

MARCHESAN, E. et al. Rice herbicide monitoring in two Brazilian rivers during the rice growing season. Scientia Agricola, Piracicaba, Brasil, v. 64, n. 2, p. 131-137, mar./apr. 2007.

MARQUES, J.F. Pesticidas na água potável: padrões de qualidade e princípio de precaução na legislação européia. Caderno $C R H$, Salvador, n. 24/25, p. 269-285, jan./dez. 1996.

MARQUES, M.N. Avaliação do impacto de agrotóxicos em áreas de proteção ambiental, pertencentes à bacia hidrográfica do Rio Ribeira de I guape, São Paulo. Uma contribuição à análise crítica da legislação sobre o padrão de potabilidade. Tese (Doutorado) - Instituto de Pesquisas Energéticas e Nucleares, São Paulo, 2005.

ORGANIZACIÓN MUNDIAL DE LA SALUD. Guías para la calidad del agua potable. V. 1: Recomendaciones. 2. Ed. Genebra: OMS, 1995.

PAUMGARTTEN, F.J.R. Risk assessment for chemical substances: the link between toxicology and public health. Cadernos de Saúde Pública, Rio de Janeiro, v. 9, n. 4, p. 439-447, out./dez. 1993.

RISSATO, S.R. et al. Determinação de pesticidas organoclorados em água de manancial, água potável e solo na região de Bauru, SP. Química Nova, v. 27, n. 5, p. 739-743, 2004

SARCINELLI, P.N. et al. Estudo dos agrotóxicos mais utilizados no país: avaliação da contaminação das águas de consumo humano nas grandes bacias hidrográficas e estratégias analíticas para o monitoramento. Relatório Final. Rio de Janeiro: Fiocruz, 2005.

SINDICATO NACIONAL DA INDÚSTRIA DE PRODUTOS PARA DEFESA AGRíCOLA. Dados de mercado. Mercado de defensivos. Câmara Temática de Insumos Agropecuários. Disponível em: http://www.sindag. com.br/upload/Vendasjaneirooutubro.ppt. Acesso em: 29 jan. 2009.

UNITED NATIONS ENVIRONMENT PROGRAMME CHEMICALS. Stockholme convention on persistent organic pollutants (POPS). 2001. Disponível em: http://www.pops.int/documents/convtext/convtext en.pdf. Acesso em: $1^{\circ}$ mai. 2007.

UNITED STATES ENVIRONMENTAL PROTECTION AGENCY. Pesticides: health and safety. Evaluating pesticides for carcinogenic potential. 2007. Disponível em: http://www.epa.gov/pesticides/health/cancerfs.htm. Acesso em: 7 mai. 2007.

Current drinking water standards. 2005A. Disponível em: http:// www.epa.gov/safewater/mcl.html. Acesso em: 6 nov. 2005.

Setting standards for safe drinking water. 2005B. Disponível em: http:// uww.epa.gov/safewater/standard/setting.html. Acesso em: 6 nov. 2005.
Edition of the drinking water standards and health advisories. EPA 822-R-04-005 Office ofWater. Washington, DC.2004. Disponívelem: http:// web.epa.state.oh.us/dsiwm/document/newsPDFs/2004dwstandards. pdf. Acesso em: 25 ago. 2006

Safety factor and other uncertainty factors in cumulative risk assessment of chemicals sharing a common mechanism of toxicity. Washington, DC. Draft of February, 2002. Disponível em: www.epa.gov/ pesticides/trac/science/cumulative_guidance.pdf. Acesso em: 30 ago. 2007.

.National primary drinking water regulations. Long Term 1 Enhanced Surface Water Treatment and Filter Backwash Rule; Proposed Rule. Part II (40 CFR, Parts 141, and 142). Washington, DC, Federal Register, Rules and regulations, Federal Register / Vol. 65, No. 69 / Monday, April 10 2000a / Proposed Rules, 2000A, p. 19046-19150.

Supplementary guidance for conducting health and risk assessment of chemical mixture. Washington, DC, August, 2000B Disponível em: http://www.epa.gov/ncea/raf/pdfs/chem_mix/chem mix 08 2001.pdf. Acesso em: $1^{\circ}$ jun. 2007.

Seminar Publication. risk assessment, management and communication of drinking water contamination. Office of Drinking Water Office of Research and Development. Office of Technology Transfer and Regulatory Support, June 1990.

UNITED STATES DEPARTMENT OF AGRICULTURE. Fact sheets. Production and Inspection. Food safety and inspection service. Disponível em: http://www.fsis.usda.gov/Fact_Sheets/Risk_Analysis/ index.asp. Acesso em 13 nov. 2007.

VEIGA, M.M. et al. Análise da contaminação dos sistemas hídricos por agrotóxicos numa pequena comunidade rural do Sudeste do Brasil. Cadernos de Saúde Pública, Rio de Janeiro, v. 22, n. 11, p. 2391-2399, nov. 2006.

WORLD HEALTH ORGANIZATION. Guidelines for drinking - water quality. Third Edition. 1 ${ }^{\text {st }}$ Addendum to vol. 1. Geneva: WHO, 2006. Disponível em: http://www.who.int/water_sanitation_health/dwq/gdwq0506.pdf. Acesso em: 29 jun. 2007.

IPCS risk assessment terminology. Harmonization Project Document n. 1. Geneva: WHO, 2004.

Principles for the assessment of risks to human health from exposure to chemicals. International Programme on Chemical Safety, Environmental Health Criteria 210. Geneva: WHO, 1999. Disponível em: http://www.inchem.org/documents/ehc/ehc/ehc210.htm. Acesso em: 5 abr. 2007.

. Assessing human health risks of chemicals: derivation of guidance values for health-based exposure limits. International Programme on Chemical Safety, Environmental Health Criteria 170. Geneva: WHO, 1994 Disponível em: http://www.inchem.org/documents/ehc/ehc/ehc170.htm. Acesso em: 5 abr. 2007. 